Pacific

Journal of

Mathematics

TRACE FORMULA FOR A SYSTEM OF PARTICLES WITH ELLIPTIC POTENTIAL

K.L. VANINSKY 


\title{
TRACE FORMULA FOR A SYSTEM OF PARTICLES WITH ELLIPTIC POTENTIAL
}

\author{
K.L. VANINSKY
}

\begin{abstract}
We consider classical particles on the line with the Weierstrass $\wp$ function as potential. This system parameterizes special solutions of the KP equation. We derive the trace formula which relates the Hamiltonian of the particle system to the residues of some Abelian differential (meromorphic one-form) on the spectral curve. Such formula is important for the construction action-angle variables and study invariant Gibbs' states.
\end{abstract}

\section{Introduction.}

The subject of this note is a system of $N$ classical particles on the line interacting with the Hamiltonian

$$
H_{N}=\sum_{n=1}^{N} \frac{p_{n}^{2}}{2}-2 \sigma^{2} \sum_{n, m=1}^{N} \wp\left(q_{n}-q_{m}\right) .
$$

The parameter ${ }^{1} \sigma=1$ corresponds to attractive particles and $\sigma=i=$ $\sqrt{-1}$ corresponds to repulsive particles. The potential is the Weierstrass $\wp$ function with real period $2 \omega$ and pure imaginary period $2 \omega^{\prime}$. The system includes the well known integrable in the sence of Liouville potentials $\sin ^{-2} x$, $\sinh ^{-2} x$ and $x^{-2}$; which correspond to various degeneration of $\wp$.

In a remarkable article Airault, McKean, Moser, [AMM], discovered a connection between particles with rational or elliptic potential and the Korteweg-de Vries equation

$$
u_{t}+\frac{3}{2} u u_{x}-\frac{1}{4} u_{x x x}=0 .
$$

Few years later Krichever, $[\mathbf{K}]$, found an isomorphism between particles with elliptic potentials and special solutions of the Kadomtzev-Petviashvilli (KP) equation

$$
\frac{3}{4} \sigma^{2} u_{y y}=\left(u_{t}+\frac{3}{2} u u_{x}-\frac{1}{4} u_{x x x}\right)_{x} .
$$

\footnotetext{
${ }^{1}$ Should not be confused with the Weierstrass $\sigma$, see Section 6 .
} 
The case $\sigma=1$ corresponds to the KP-2 equation and $\sigma=i$ to KP-1. The KP equation arises as a compatibility condition for the zero curvature representation

$$
\left[\sigma \partial_{y}-L_{2}, \partial_{t}-L_{3}\right]=0
$$

where

$$
\begin{aligned}
& L_{2}=\partial_{x}^{2}-u, \\
& L_{3}=\partial_{x}^{3}-\frac{3}{2} u \partial_{x}-w .
\end{aligned}
$$

Any such solution is associated with a spectral curve $\Gamma_{N}$ of genus $N$, defined by

$$
R_{N}(k, z)=\sum_{n=0}^{N} r_{n}(z) k^{n}=\operatorname{det}(\tilde{L}+2 k),
$$

where $\tilde{L}$ is a $N \times N$ matrix which depends on $q, p$ and $z$. The functions $r_{n}(z)$ are elliptic, so the curve $\Gamma_{N}$ is an $N$-sheeted covering of the elliptic curve. The matrix $\tilde{L}$ has a simple pole above $z=0$ and can be expanded in powers of $z$

$$
\tilde{L}=\frac{1}{z} L^{(-1)}+O(1),
$$

where $L_{n m}=-2\left(1-\delta_{n m}\right)$ is a constant matrix. This "zero order" approximation provides all the information needed to solve the direct spectral problem and obtain a formula for the solution in terms of Riemann theta functions, see $[\mathbf{K}]$.

In this note we address a different question. Is there a formula of the type

$$
H_{N}=\sum_{\alpha=2}^{N} I_{\alpha}^{\prime},
$$

where $I_{\alpha}^{\prime}$ are parameters of the Riemann surface associated with the system? We give an affirmative answer to this question here. The formula is needed, see [MCV1, MCV2], to express the canonical measure as

$$
e^{-H} d v o l=e^{-H} \prod d I d \phi=e^{-\sum I^{\prime}} \prod d I d \phi,
$$

where $d v o l$ is produced from the basic symplectic structure $\Omega=d p \wedge d q$ and $I$ 's and $\phi$ 's are classical action-angle variables constructed from $\Omega$. After this is done one can try to compute the partition function, [V1].

Now analysis of the direct spectral problem requires a "second order" approximation

$$
\tilde{L}=\frac{1}{z} L^{(-1)}+L^{(0)}+z L^{(1)}+O\left(z^{2}\right)
$$


Such an approximation provides the coefficients $k_{1}^{(0)}$ and $k_{1}^{(1)}$ (Theorem 9) for the expansion of the function $k$

$$
k_{1}(z)=\frac{N-1}{z}+k_{1}^{(0)}+z k_{1}^{(1)}+O\left(z^{2}\right)
$$

on the "upper" sheet of the curve. The desired formula can be easily obtained by a simple application of Cauchy's theorem. Moreover, in the repulsive case the parameters $I_{\alpha}^{\prime}$ are real for all configurations of particles. In order to prove this we show that in the expansion

$$
k_{\alpha}(z)=-\frac{1}{z}+k_{\alpha}^{(0)}+O(z), \quad \alpha=2, \ldots, N,
$$

the coefficients $k_{\alpha}^{(0)}$ are distinct for all $\alpha=2, \cdots, N$ and a generic configuration of particles. That much information can be obtained by perturbation techniques on "lower" sheets.

Presumably $I_{\alpha}^{\prime}$ are moduli of the corresponding $N$-sheeted covers and the actions relative to some symplectic structure $\Omega^{\prime}$ on the phase space, but this is not proved. Compare [V2] for the case of the cubic Schrödinger curves. We will return to this issue elsewhere.

\section{Elliptic solutions of the KP hierarchy.}

Consider the $N$ particle Hamiltonian on the line

$$
H_{N}=\sum_{n=1}^{N} \frac{p_{n}^{2}}{2}-2 \sigma^{2} \sum_{n, m=1}^{N} \wp\left(q_{n}-q_{m}\right) .
$$

The Hamiltonian produces a system of first order equations of motion

$$
\begin{array}{ll}
\dot{q}_{n}=\frac{\partial H}{\partial p_{n}}, & n=1, \ldots, N, \\
\dot{p}_{n}=-\frac{\partial H}{\partial q_{n}}=4 \sigma^{2} \sum_{m \neq n} \wp^{\prime}\left(q_{n}-q_{m}\right), & n=1, \ldots, N .
\end{array}
$$

The system can be written in the form

$$
\ddot{q}_{n}=4 \sigma^{2} \sum_{m \neq n} \wp^{\prime}\left(q_{n}-q_{m}\right), \quad n=1, \ldots, N
$$

The key step in the embedding of the particle system into the elliptic solutions of the KP equation is the following theorem: 
Theorem 1 ([K]). The equations

$$
\begin{gathered}
{\left[\sigma \partial_{y}-\partial_{x}^{2}+2 \sum_{n=1}^{N} \wp\left(x-q_{n}(y)\right)\right] \psi=0,} \\
\psi^{\dagger}\left[\sigma \partial_{y}-\partial_{x}^{2}+2 \sum_{n=1}^{n} \wp\left(x-q_{n}(y)\right)\right]=0
\end{gathered}
$$

have solutions of the form

$$
\begin{aligned}
\psi(x, y, k, z) & =\sum_{n=1}^{N} a_{n}(y, k, z) \Phi\left(x-q_{n}, z\right) e^{k x+\sigma^{-1} k^{2} y} \\
\psi^{\dagger}(x, y, k, z) & =\sum_{n=1}^{N} a_{n}^{\dagger}(y, k, z) \Phi\left(-x+q_{n}, z\right) e^{-k x-\sigma^{-1} k^{2} y},
\end{aligned}
$$

where $^{2} \Phi(x, z)=\frac{\sigma(z-x)}{\sigma(z) \sigma(x)} e^{\zeta(z) x}$, if and only if $q_{n}(y)$ satisfy the system of equations (1).

The proof is obtained by requiring that singularities of the form $\left(x-q_{n}\right)^{-2}$ and $\left(x-q_{n}\right)^{-1}$ vanish. This condition can be written in a compact form with the aid of $N \times N$ matrices $L$ and $M$

$$
\begin{aligned}
L_{n m} & =\sigma p_{n} \delta_{n m}+2 \Phi\left(q_{n}-q_{m}, z\right)\left(1-\delta_{n m}\right), \\
M_{n m} & =\left(-\wp(z)+2 \sum_{s \neq n} \wp\left(q_{n}-q_{s}\right)\right) \delta_{n m}+2 \Phi^{\prime}\left(q_{n}-q_{m}, z\right)\left(1-\delta_{n m}\right) .
\end{aligned}
$$

Lemma $2([\mathbf{K}])$. The vectors $a(y, k, z)$ and $a^{\dagger}(y, k, z)$ satisfy the equations

$$
(L+2 k) a=0 \quad\left(\sigma \partial_{y}+M\right) a=0
$$

and

$$
a^{\dagger}(L+2 k)=0 \quad a^{\dagger}\left(\sigma \partial_{y}+M\right)=0 .
$$

These equations determine the curve $\Gamma_{N}$ which is the subject of the next section.

\section{Riemann surface.}

The matrix $L$ can be simplified using a gauge transformation

$$
L=G \tilde{L} G^{-1},
$$

where $G_{n m}=e^{\zeta(z) q_{n}} \delta_{n m}$. Then

$$
\tilde{L}_{n m}=\sigma p_{n} \delta_{n m}+2 \Phi_{0}\left(q_{n}-q_{m}, z\right)\left(1-\delta_{n m}\right)
$$

\footnotetext{
${ }^{2} \sigma(z)$ denotes the Weierstrass function.
} 
and

$$
\Phi_{0}(x, z)=\frac{\sigma(z-x)}{\sigma(z) \sigma(x)} .
$$

The existence of a nontrivial vector $a:(\tilde{L}+2 k) a=0$ implies that $R_{N}(k, z)=$ $\operatorname{det}(\tilde{L}+2 k)$ vanishes and this condition determines the curve. We denote by $P, Q$, etc., points $(k, z)$ on the curve.

Lemma $3([\mathbf{K}])$. The determinant $R_{N}(k, z)$ can be written in the form

$$
R_{N}(k, z)=\sum_{n=0}^{N} r_{n}(z) k^{n},
$$

where $r_{n}(z)$ are elliptic functions of $z$.

The curve $\Gamma_{N}$ is an $N$ sheeted covering of the elliptic curve. The next lemma describes symmetries of the curve.

Lemma 4. (i) $\sigma=1$. The curve $\Gamma_{N}$ admits the antiholomorphic involution

$$
\tau_{1}: \quad(k, z) \rightarrow(\bar{k}, \bar{z}) .
$$

(ii) $\sigma=i$. The curve $\Gamma_{N}$ admits the antiholomorphic involution

$$
\tau_{i}: \quad(k, z) \rightarrow(-\bar{k},-\bar{z}) .
$$

Proof. (i) For rectangular lattice $\overline{\sigma(z)}=\sigma(\bar{z})$ and $\overline{\Phi_{0}(x, z)}=\Phi_{0}(\bar{x}, \bar{z})$. Therefore $\overline{R_{N}(k, z)}=R_{N}(\bar{k}, \bar{z})$.

(ii) Note first $\Phi_{0}(x,-z)=-\Phi_{0}(-x, z)$ and $\overline{i p+2 k}=-(i p+2(-\bar{k}))$. Therefore $\overline{R_{N}(k, z)}=(-1)^{N} R_{N}(-\bar{k},-\bar{z})$. The proof is finished.

The function $\Phi_{0}(x, z)$ has a simple pole at $z=0$ and can be expanded in powers of $z$

$$
\Phi_{0}(x, z)=-\frac{1}{z}+\zeta(x)+\frac{1}{2}\left(\wp(x)-\zeta^{2}(x)\right) z+O\left(z^{2}\right) .
$$

Therefore $^{3}$,

$$
\tilde{L}(p, q, z)=\frac{1}{z} L^{(-1)}(p, q)+L^{(0)}(p, q)+z L^{(1)}(p, q)+O\left(z^{2}\right),
$$

where

$$
\begin{aligned}
L_{n m}^{(-1)} & =-2\left(1-\delta_{n m}\right), \\
L_{n m}^{(0)} & =\sigma p_{n} \delta_{n m}+2 \zeta\left(q_{n}-q_{m}\right)\left(1-\delta_{n m}\right), \\
L_{n m}^{(1)} & =\left(\wp-\zeta^{2}\right)\left(q_{n}-q_{m}\right)\left(1-\delta_{n m}\right) .
\end{aligned}
$$

The matrix $L^{(-1)}$ is a constant matrix. Its spectrum and eigenvectors can be easily computed.

\footnotetext{
${ }^{3}$ We omit ${ }^{\sim}$ above $L$ and $a$ to simplify the notations.
} 
Let

$$
a_{\alpha}=\left(\begin{array}{c}
1 \\
e^{i \beta_{\alpha}} \\
\vdots \\
e^{i \beta_{\alpha}(N-1)}
\end{array}\right) \quad \text { and } \quad \beta_{\alpha}=\frac{2 \pi}{N}(\alpha-1), \quad \alpha=1, \ldots, N
$$

Then

$$
\left(L^{(-1)}+2 k_{\alpha}\right) a_{\alpha}=0
$$

with $k_{\alpha}=-1$ for $\alpha=2, \ldots, N$ and $k_{1}=N-1$.

The necessary information about the curve is obtained by perturbation of this trivial case. The eigenvectors $a_{\alpha}(z)$ and eigenvalues $k_{\alpha}(z)$ can be expanded in power series in $z$

$$
\begin{array}{ll}
a_{\alpha}(z)=a_{\alpha}^{(0)}+a_{\alpha}^{(1)} z+a_{\alpha}^{(2)} z^{2}+\cdots, & a_{\alpha}^{(0)}=a_{\alpha}, \\
k_{\alpha}(z)=\frac{1}{z} k_{\alpha}^{(-1)}+k_{\alpha}^{(0)}+k_{\alpha}^{(1)} z+\cdots, & k_{\alpha}^{(-1)}=k_{\alpha} .
\end{array}
$$

The index " $\alpha$ " labels the sheets of the curve $\Gamma_{N}$. We call the sheet $\alpha=1$ the "upper" sheet. In fact, the upper sheet is distinguished by $k^{(-1)}$, the leading term of the asymptotics. The "lower" sheets $(\alpha \geq 2)$ are distinguished for generic configuration of particles by different values of $k^{(0)}$ since all $k^{(-1)}=$ -1 . This is proved in Lemma 5. The proof of Lemma 6 shows that all leading terms $a_{\alpha}^{(0)}$ are distinct. This implies that all "lower" sheets can be indexed according to these asymptotics and $a_{\alpha}^{(0)}=a_{\alpha}$.

Lemma 5. For generic configuration of particles $k^{(0)}$ are distinct.

Proof. To prove the statement we need first order perturbation theory for multiple eigenvalues.

We choose $N-1$ vectors $e^{(0)}$ in the subspace generated by $a_{\alpha}^{(0)}, \alpha=$ $2, \ldots, N$;

$$
e^{(0)}=\sum_{\alpha=2}^{N} \eta_{\alpha} a_{\alpha}^{(0)},
$$

where the $\eta$ 's depend on $e^{(0)}$ and are such that

$$
(\tilde{L}(z)+2 k(z)) e_{\gamma}(z)=0 .
$$

$L(z), k_{\gamma}(z), e_{\gamma}(z)$ can be expanded in integer powers of $z$

$$
\begin{aligned}
& k_{\gamma}(z)=\frac{1}{z} k_{\gamma}^{(-1)}+k_{\gamma}^{(0)}+z k_{\gamma}^{(1)}+\cdots \\
& e_{\gamma}(z)=e_{\gamma}^{(0)}+z e_{\gamma}^{(1)}+\cdots
\end{aligned}
$$


Now we collect terms in the identity

$$
\left[\frac{1}{z} L^{(-1)}+L^{(0)}+\cdots+\frac{2}{z} k^{(-1)}+2 k^{(0)}+\cdots\right]\left[e^{(0)}+z e^{(1)}+\cdots\right]=0 .
$$

Terms in $z^{0}$ produce

$$
L^{(-1)} e^{(1)}+L^{(0)} e^{(0)}+2 k^{(-1)} e^{(1)}+2 k^{(0)} e^{(0)}=0,
$$

and

$$
\left(L^{(-1)} e^{(1)}, a_{\alpha}^{(0)}\right)+\left(L^{(0)} e^{(0)}, a_{\alpha}^{(0)}\right)+2 k^{(-1)}\left(e^{(1)}, a_{\alpha}^{(0)}\right)+2 k^{(0)}\left(e^{(0)}, a_{\alpha}^{(0)}\right)=0 .
$$

Using the selfadjointness of $L^{(-1)}$

$$
\left(L^{(-1)} e^{(1)}, a_{\alpha}^{(0)}\right)=\left(e^{(1)}, L^{(-1)} a_{\alpha}^{(0)}\right)=-2 k^{(-1)}\left(e^{(1)}, a_{\alpha}^{(0)}\right) .
$$

Therefore

$$
\left(L^{(0)} e^{(0)}, a_{\alpha}^{(0)}\right)=-2 k^{(0)}\left(e^{(0)}, a_{\alpha}^{(0)}\right)
$$

or

$$
\sum_{\alpha=2}^{N} \eta_{\alpha}\left(L^{(0)} a_{\alpha}^{(0)}, a_{\alpha^{\prime}}^{(0)}\right)=-2 k^{(0)} \eta_{\alpha^{\prime}} .
$$

The eigenvalues $-2 k^{(0)}$ of the matrix $\left(L^{(0)} a_{\alpha}^{(0)}, a_{\alpha^{\prime}}^{(0)}\right), \alpha, \alpha^{\prime}=2, \ldots, N$; are distinct for generic configuration of particles.

Lemma 6. For all configurations of particles the "zero" order approximations

$$
\tilde{a}^{(0)}=\left(\begin{array}{c}
\tilde{a}^{(0)}(1) \\
\vdots \\
\tilde{a}^{(0)}(N)
\end{array}\right)
$$

of the eigenvectors $\tilde{a}_{\alpha}(z)=\tilde{a}_{\alpha}^{(0)}+\tilde{a}_{\alpha}^{(1)} z+\cdots$ of the spectral problem $(\tilde{L}+$ $2 k) \tilde{a}=0$ normalized by the condition $\tilde{a}^{(0)}(1)=1$ are given by the formula

$$
\tilde{a}_{\alpha}^{(0)}=\left(\begin{array}{c}
1 \\
e^{i \beta_{\alpha}} \\
\vdots \\
e^{i \beta_{\alpha}(N-1)}
\end{array}\right) \quad \text { and } \beta_{\alpha}=\frac{2 \pi}{N}(\alpha-1), \quad \alpha=1, \ldots, N \text {. }
$$

Proof. If $\mathcal{A}$ as $n \times n$ matrix then $\mathcal{A} \mathcal{A}^{\wedge}=\operatorname{det} \mathcal{A} I$, where $\mathcal{A}^{\wedge}$ is the matrix which consists of auxiliary minors of $\mathcal{A}$, see also [KNS]. For any column $r=1, \ldots, N$

$$
\tilde{a}(p)=\frac{[\tilde{L}+2 k]_{p r}^{\wedge}}{[\tilde{L}+2 k]_{1 r}^{\wedge}}
$$


in the vicinity of $z=0^{4}$.

We know that $k(z)=-\frac{1}{z}+k^{(0)}+\cdots$ and

$$
\tilde{L}=-\frac{2}{z}[E-I]+\tilde{L}^{(0)}+\cdots, \quad E_{n m}=1 .
$$

Then

$$
[\tilde{L}+2 k]_{p r}^{\wedge}=\left[-\frac{2 E}{z}+\left[\tilde{L}^{(0)}+2 k^{(0)}\right]+\cdots\right]_{p r}^{\wedge} .
$$

Since $\operatorname{rank} E=1$ we have

$$
[\tilde{L}+2 k]_{p r}^{\wedge}=-\frac{2}{z} \sum_{s=1}^{N}\left[\tilde{L}^{(0)}+2 k^{(0)}\right]_{\hat{s} p r}^{\wedge}+\cdots
$$

where the subscript $\hat{s}$ means that the $s$-th column is replaced by $\left(\begin{array}{c}1 \\ \vdots \\ 1\end{array}\right)$.

For a generic configuration $k^{(0)}$ are distinct on all sheets of the curve. This and the formula for $\tilde{a}(p)$ imply that all $\tilde{a}^{(0)}$ are also distinct and therefore match $\tilde{a}_{\alpha}^{(0)}$. Since all $\tilde{a}_{\alpha}^{(0)}$ are fixed the statement is true for all configurations of particles.

The following two lemmas are simple consequences of the discussion above:

Lemma $7([\mathbf{K}])$. The determinant $R_{N}(k, z)$ can be written in the form $R_{N}(k, z)=2^{N}\left(k-\left(\frac{N-1}{z}+k_{1}^{(0)}+\cdots\right)\right) \prod_{\alpha=2}^{N}\left(k-\left(-\frac{1}{z}+k_{\alpha}^{(0)}+\cdots\right)\right)$.

The genus of the curve $\Gamma_{N}$ can be easily computed using the RiemannHurwitz formula.

Lemma 8 ([K, KBBT $])$. (i) The elliptic case: $2 \omega, 2 \omega^{\prime}<\infty$. For generic configuration of particles the genus of the curve $\Gamma_{N}$ is $N$.

(ii) The rational case: $2 \omega, 2 \omega^{\prime}=\infty$. The genus of the curve $\Gamma_{N}$ is 0 .

\section{Asymptotics for $k(z)$.}

The main result of this section is the following:

Theorem 9. On the "upper" sheet for $k_{1}(z)$ the following asymptotics hold

$$
k_{1}(z)=\frac{N-1}{z}-\frac{\sigma P_{N}}{2 N}+z\left(\frac{\sigma^{2} H_{N}}{2 N^{2}}-\frac{\sigma^{2} P_{N}^{2}}{4 N^{3}}\right)+O\left(z^{2}\right),
$$

\footnotetext{
${ }^{4}$ Note that $\tilde{a}$ is not a function on the curve, since the entries of the matrix $\tilde{L}+2 k$ are not elliptic functions.
} 
where

$$
H_{N}=K_{N}-\sigma^{2} V_{N}=\sum_{n=1}^{N} \frac{p_{n}^{2}}{2}-2 \sigma^{2} \sum_{n, m=1}^{N} \wp\left(q_{n}-q_{m}\right),
$$

and

$$
P_{N}=\sum_{n=1}^{N} p_{n}
$$

To prove the theorem we need the following lemma which is the second order perturbation theory of simple eigenvalues adapted to our considerations.

Lemma 10. The following identities hold

$$
\begin{aligned}
& 2 k^{(0)}=-\left(L^{(0)} a^{(0)}, a^{(0)}\right), \\
& 2 k^{(1)}=\frac{1}{2 N} \sum_{\alpha=2}^{N}\left(L^{(0)} a_{\alpha}^{(0)}, a^{(0)}\right) \times\left(L^{(0)} a^{(0)}, a_{\alpha}^{(0)}\right)-\left(L^{(1)} a^{(0)}, a^{(0)}\right),
\end{aligned}
$$

where

$$
(f, g)=\frac{1}{N} \sum_{n=1}^{N} f_{n} \bar{g}_{n}
$$

for any $f, g \in \mathbb{C}^{N}$.

Proof. We start with the identity

$$
\begin{aligned}
\left(\frac{1}{z} L^{(-1)}+L^{(-0)}\right. & +z L^{(1)}+\cdots \\
+ & \left.\frac{2 k^{(-1)}}{z}+2 k^{(0)}+2 k^{(1)}+\cdots\right)\left(a^{(0)}+z a^{(1)}+\cdots\right)=0 .
\end{aligned}
$$

Collecting the terms in $z^{-1}$

$$
\left(L^{(-1)}+2 k^{(-1)}\right) a^{(0)}=0 .
$$

Note,

$$
a^{(0)}=a_{1}^{(0)}=\left(\begin{array}{c}
1 \\
1 \\
\vdots \\
1
\end{array}\right) .
$$

Therefore $k^{(-1)}=N-1$, as it has to be.

Now we collect terms with $z^{0}$

$$
L^{(-1)} a^{(1)}+L^{(0)} a^{(0)}+2 k^{(-1)} a^{(1)}+2 k^{(0)} a^{(0)}=0
$$


and

(3)

$\left(L^{(-1)} a^{(1)}, a^{(0)}\right)+\left(L^{(0)} a^{(0)}, a^{(0)}\right)+\left(2 k^{(-1)} a^{(1)}, a^{(0)}\right)+\left(2 k^{(0)} a^{(0)}, a^{(0)}\right)=0$.

Note

$$
\left(L^{(-1)} a^{(1)}, a^{(0)}\right)=\left(a^{(1)}, L^{(-1)} a^{(0)}\right)=\left(a^{(1)},-2 k^{(-1)} a^{(0)}\right),
$$

which implies that the two terms in (3) cancel each other. From the definition $\left(a^{(0)}, a^{(0)}\right)=1$ we obtain the first statement of the lemma.

Now we derive the formulas for $a^{(1)}$ on the "upper" sheet, which will be useful later on. Multiply (2) by $a_{\alpha}^{(0)}, \alpha=2, \ldots, N$,

$$
\left(L^{(-1)} a^{(1)}, a_{\alpha}^{(0)}\right)+\left(L^{(0)} a^{(0)}, a_{\alpha}^{(0)}\right)+\left(2 k^{(-1)} a^{(1)}, a_{\alpha}^{(0)}\right)+\left(2 k^{(0)} a^{(0)}, a_{\alpha}^{(0)}\right)=0 .
$$

The last term vanishes due to the orthogonality of eigenvectors corresponding different eigenvalues. Similar to (4)

$$
\left(L^{(-1)} a^{(1)}, a_{\alpha}^{(0)}\right)=\left(a^{(1)}, L^{(-1)} a_{\alpha}^{(0)}\right)=2\left(a^{(1)}, a_{\alpha}^{(0)}\right)
$$

due to

$$
\left(\frac{1}{z} L^{(-1)}+2\left(-\frac{1}{z}\right)\right) a_{\alpha}^{(0)}=0, \quad \alpha=2, \ldots, N .
$$

Therefore

$$
2\left(a^{(1)}, a_{\alpha}^{(0)}\right)+\left(L^{(0)} a^{(0)}, a_{\alpha}^{(0)}\right)+2(N-1)\left(a^{(1)}, a_{\alpha}^{(0)}\right)=0
$$

and

$$
\left(a^{(1)}, a_{\alpha}^{(0)}\right)=-\frac{1}{2 N}\left(L^{(0)} a^{(0)}, a_{\alpha}^{(0)}\right), \quad \alpha=2, \ldots, N .
$$

The condition $\|a(z)\|^{2}=1+O\left(z^{2}\right)$ implies

$$
\begin{aligned}
1=(a(z), a(z)) & =\left(a^{(0)}+z a^{(1)}+\cdots, a^{(0)}+z a^{(1)}+\cdots\right) \\
& =\left(a^{(0)}, a^{(0)}\right)+z\left[\left(a^{(0)}, a^{(1)}\right)+\left(a^{(1)}, a^{(0)}\right)\right]+\cdots .
\end{aligned}
$$

and $\left(a^{(1)}, a^{(0)}\right)=0$. Therefore

$$
a^{(1)}=\sum_{\alpha=2}^{N} a_{\alpha}^{(0)}\left(a^{(1)}, a_{\alpha}^{(0)}\right)=-\frac{1}{2 N} \sum_{\alpha=2}^{N} a_{\alpha}^{(0)}\left(L^{(0)} a^{(0)}, a_{\alpha}^{(0)}\right) .
$$

In order to prove the second formula of the lemma we collect terms with $z^{1}$

$$
L^{(-1)} a^{(2)}+L^{(0)} a^{(1)}+L^{(1)} a^{(0)}+2 k^{(-1)} a^{(2)}+2 k^{(0)} a^{(1)}+2 k^{(1)} a^{(0)}=0
$$


and

$$
\begin{aligned}
& \left(L^{(-1)} a^{(2)}, a^{(0)}\right)+\left(L^{(0)} a^{(1)}, a^{(0)}\right)+\left(L^{(1)} a^{(0)}, a^{(0)}\right) \\
& \quad+2 k^{(-1)}\left(a^{(2)}, a^{(0)}\right)+2 k^{(0)}\left(a^{(1)}, a^{(0)}\right)+2 k^{(1)}\left(a^{(0)}, a^{(0)}\right)=0 .
\end{aligned}
$$

Note

$$
\left(L^{(-1)} a^{(2)}, a^{(0)}\right)=\left(a^{(2)}, L^{(-1)} a^{(0)}\right)=-2 k^{(-1)}\left(a^{(2)}, a^{(0)}\right)
$$

and two of the terms in the formula (6) vanish. Using the normalization conditions $\left(a^{(0)}, a^{(0)}\right)=1$ and $\left(a^{(1)}, a^{(0)}\right)=0$ we obtain

$$
2 k^{(1)}=-\left(L^{(0)} a^{(1)}, a^{(0)}\right)-\left(L^{(1)} a^{(0)}, a^{(0)}\right) .
$$

Using the formula for $a^{(1)}$, we finally have

$$
2 k^{(1)}=\frac{1}{2 N} \sum_{\alpha=2}^{N}\left(L^{(0)} a_{\alpha}^{(0)}, a^{(0)}\right) \times\left(L^{(0)} a^{(0)}, a_{\alpha}^{(0)}\right)-\left(L^{(1)} a^{(0)}, a^{(0)}\right) .
$$

The lemma is proved.

Now we use the lemma to compute the coefficients $k^{(0)}$ and $k^{(1)}$. Note, first, that $L^{(0)}$ can be split into two parts $L^{(0)}=\sigma A+B$, where

$$
A_{m n}=p_{n} \delta_{n m} \quad \text { and } \quad B_{n m}=2 \zeta\left(q_{n}-q_{m}\right)\left(1-\delta_{n m}\right) .
$$

The matrix $A$ is symmetric and $B$ is skew-symmetric

$$
\begin{aligned}
2 k^{(0)} & =-\left(L^{(0)} a^{(0)}, a^{(0)}\right)=-\sigma\left(A a^{(0)}, a^{(0)}\right)-\left(B a^{(0)}, a^{(0)}\right) \\
& =-\frac{\sigma P_{N}}{N} .
\end{aligned}
$$

The term with $B$ vanishes due to skew-symmetry.

The computation of the next term $k^{(1)}$ is much more involved. Using the decomposition for $L^{(0)}$ we have

$$
\begin{aligned}
2 k^{(1)}= & \frac{1}{2 N} \sum_{\alpha=2}^{N}\left[\sigma\left(A a_{\alpha}^{(0)}, a^{(0)}\right)+\left(B a_{\alpha}^{(0)}, a^{(0)}\right)\right] \\
& \times\left[\sigma\left(A a^{(0)}, a_{\alpha}^{(0)}\right)+\left(B a^{(0)}, a_{\alpha}^{(0)}\right)\right]-\left(L^{(1)} a^{(0)}, a^{(0)}\right)=I+I I .
\end{aligned}
$$

Step 1. Our goal is to evaluate the first term I. Arguments for the attractive and the repulsive case are different and we consider these two cases separately. 
Repulsive case. $\sigma=i$. Using the symmetry of $A$ and skew-symmetry of $B$,

$$
\begin{aligned}
& \frac{1}{2 N} \sum_{\alpha=2}^{N}\left[i\left(A a_{\alpha}^{(0)}, a^{(0)}\right)+\left(B a_{\alpha}^{(0)}, a^{(0)}\right)\right] \times\left[i\left(A a^{(0)}, a_{\alpha}^{(0)}\right)+\left(B a^{(0)}, a_{\alpha}^{(0)}\right)\right] \\
& =-\frac{1}{2 N} \sum_{\alpha=2}^{N}\left|i\left(A a^{(0)}, a_{\alpha}^{(0)}\right)+\left(B a^{(0)}, a_{\alpha}^{(0)}\right)\right|^{2} \\
& =-\frac{1}{2 N} \sum_{\alpha=1}^{N}\left|i\left(A a^{(0)}, a_{\alpha}^{(0)}\right)+\left(B a^{(0)}, a_{\alpha}^{(0)}\right)\right|^{2}+\frac{1}{2 N}\left|\left(A a^{(0)}, a^{(0)}\right)\right|^{2} .
\end{aligned}
$$

The last term can be easily estimated

$$
\frac{1}{2 N}\left|\left(A a^{(0)}, a^{(0)}\right)\right|^{2}=\frac{1}{2 N^{3}} P_{N}^{2} .
$$

To estimate the first term, we introduce $\alpha^{\prime}$ such that

$$
\alpha^{\prime}=N-\alpha+2 \text {. }
$$

Then

$$
\beta_{\alpha^{\prime}}=\frac{2 \pi}{N}\left(\alpha^{\prime}-1\right)=\frac{2 \pi}{N}(N-\alpha+1)
$$

and

$$
\beta_{\alpha}+\beta_{\alpha^{\prime}} \equiv 0 \quad(\bmod 2 \pi)
$$

Now

$$
\begin{aligned}
- & \frac{1}{2 N} \sum_{\alpha=1}^{N}\left|i\left(A a^{(0)}, a_{\alpha}^{(0)}\right)+\left(B a^{(0)}, a_{\alpha}^{(0)}\right)\right|^{2} \\
= & -\frac{1}{4 N} \sum_{\alpha=1}^{N}\left|i\left(A a^{(0)}, a_{\alpha}^{(0)}\right)+\left(B a^{(0)}, a_{\alpha}^{(0)}\right)\right|^{2} \\
& +\left|\overline{i\left(A a^{(0)}, a_{\alpha^{\prime}}^{(0)}\right)+\left(B a^{(0)}, a_{\alpha^{\prime}}^{(0)}\right)}\right|^{2} \\
= & -\frac{1}{4 N} \sum_{\alpha=1}^{N}\left|i\left(A a^{(0)}, a_{\alpha}^{(0)}\right)+\left(B a^{(0)}, a_{\alpha}^{(0)}\right)\right|^{2} \\
& +\left|-i\left(A a^{(0)}, a_{\alpha}^{(0)}\right)+\left(B a^{(0)}, a_{\alpha}^{(0)}\right)\right|^{2} .
\end{aligned}
$$

Using the identity $|a+b|^{2}+|a-b|^{2}=2|a|^{2}+2|b|^{2}$ we obtain

$$
I=-\frac{1}{2 N} \sum_{\alpha=1}^{N}\left|\left(A a^{(0)}, a_{\alpha}^{(0)}\right)\right|^{2}+\left|\left(B a^{(0)}, a_{\alpha}^{(0)}\right)\right|^{2}+\frac{1}{2 N^{3}} P_{N}^{2} .
$$


Attractive case. $\sigma=1$. Again using properties of $A$ and $B$ we have

$$
\begin{aligned}
& \frac{1}{2 N} \sum_{\alpha=2}^{N}\left[\left(A a_{\alpha}^{(0)}, a^{(0)}\right)+\left(B a_{\alpha}^{(0)}, a^{(0)}\right)\right] \times\left[\left(A a^{(0)}, a_{\alpha}^{(0)}\right)+\left(B a^{(0)}, a_{\alpha}^{(0)}\right)\right] \\
& =\frac{1}{2 N} \sum_{\alpha=2}^{N}\left[\overline{\left(A a^{(0)}, a_{\alpha}^{(0)}\right)}-\overline{\left(B a^{(0)}, a_{\alpha}^{(0)}\right)}\right] \times\left[\left(A a^{(0)}, a_{\alpha}^{(0)}\right)+\left(B a^{(0)}, a_{\alpha}^{(0)}\right)\right] \\
& =\frac{1}{2 N} \sum_{\alpha=2}^{N}\left|\left(A a^{(0)}, a_{\alpha}^{(0)}\right)\right|^{2}-\left|\left(B a^{(0)}, a_{\alpha}^{(0)}\right)\right|^{2} \\
& \quad+2 i \frac{1}{2 N} \Im \sum_{\alpha=2}^{N} \overline{\left(A a^{(0)}, a_{\alpha}^{(0)}\right)}\left(B a^{(0)}, a_{\alpha}^{(0)}\right) .
\end{aligned}
$$

The second sum vanishes. Indeed,

$$
\begin{aligned}
& 2 \Im \sum_{\alpha=2}^{N} \overline{\left(A a^{(0)}, a_{\alpha}^{(0)}\right)}\left(B a^{(0)}, a_{\alpha}^{(0)}\right) \\
& =\Im \sum_{\alpha=1}^{N} \overline{\left(A a^{(0)}, a_{\alpha}^{(0)}\right)}\left(B a^{(0)}, a_{\alpha}^{(0)}\right)+\overline{\left(A a^{(0)}, a_{\alpha^{\prime}}^{(0)}\right)}\left(B a^{(0)}, a_{\alpha^{\prime}}^{(0)}\right) \\
& =\Im \sum_{\alpha=1}^{N} \overline{\left(A a^{(0)}, a_{\alpha}^{(0)}\right)}\left(B a^{(0)}, a_{\alpha}^{(0)}\right)+\left(A a^{(0)}, a_{\alpha}^{(0)}\right) \overline{\left(B a^{(0)}, a_{\alpha}^{(0)}\right)} \\
& =0 .
\end{aligned}
$$

Therefore,

$$
I=-\frac{1}{2 N} \sum_{\alpha=1}^{N}-\left|\left(A a^{(0)}, a_{\alpha}^{(0)}\right)\right|^{2}+\left|\left(B a^{(0)}, a_{\alpha}^{(0)}\right)\right|^{2}-\frac{1}{2 N^{3}} P_{N}^{2} .
$$

Combining the results for two cases $\sigma^{2}= \pm 1$, we obtain

$$
I=-\frac{1}{2 N} \sum_{\alpha=1}^{N}-\sigma^{2}\left|\left(A a^{(0)}, a_{\alpha}^{(0)}\right)\right|^{2}+\left|\left(B a^{(0)}, a_{\alpha}^{(0)}\right)\right|^{2}-\frac{\sigma^{2}}{2 N^{3}} P_{N}^{2} .
$$

Step 2. Now we estimate $\sum_{\alpha=1}^{N}\left|\left(A a^{(0)}, a_{\alpha}^{(0)}\right)\right|^{2}$ and $\left|\left(B a^{(0)}, a_{\alpha}^{(0)}\right)\right|^{2}$. To do this we introduce for two polynomials

$$
\mathcal{P}(z)=\sum_{k=1}^{N} p_{k} z^{k}, \quad \mathcal{Q}(z)=\sum_{k=1}^{N} q_{k} z^{k},
$$


the inner product

$$
\langle\mathcal{P}, \mathcal{Q}\rangle \equiv \sum_{\alpha=1}^{N} \mathcal{P}\left(e^{i \beta_{\alpha}}\right) \overline{\mathcal{Q}\left(e^{i \beta_{\alpha}}\right)}
$$

It is easy to see that

$$
\langle\mathcal{P}, \mathcal{Q}\rangle=N \sum_{k=1}^{N} p_{k} \overline{q_{k}}
$$

Therefore

$$
\begin{aligned}
& \sum_{\alpha=1}^{N}\left|\left(A a^{(0)}, a_{\alpha}^{(0)}\right)\right|^{2}=\sum_{\alpha=1}^{N}\left|\frac{1}{N} \sum_{n=1}^{N} p_{n} e^{-i \beta_{\alpha}(n-1)}\right|^{2} \\
& =\frac{1}{N^{2}}\left\langle\sum_{n=1}^{N} p_{n} z^{n}, \sum_{n=1}^{N} p_{n} z^{n}\right\rangle=\frac{1}{N} \sum_{n=1}^{N} p_{n}^{2} .
\end{aligned}
$$

Similarly,

$$
\sum_{\alpha=1}^{N}\left|\left(B a^{(0)}, a_{\alpha}^{(0)}\right)\right|^{2}=\frac{1}{N} \sum_{n=1}^{N}\left(b_{n}\right)^{2}, \quad b_{n}=\sum_{s=1}^{N} b_{n s} .
$$

Finally,

$$
\begin{aligned}
I & =\frac{\sigma^{2}}{2 N^{2}} \sum_{n=1}^{N} p_{n}^{2}-\frac{1}{2 N^{2}} \sum_{n=1}^{N} b_{n}^{2}-\frac{\sigma^{2}}{2 N^{3}} P_{N}^{2} \\
& =\frac{\sigma^{2}}{N^{2}} K_{N}-\frac{1}{2 N^{2}} \sum_{n=1}^{N} b_{n}^{2}-\frac{\sigma^{2}}{2 N^{3}} P_{N}^{2} .
\end{aligned}
$$

Step 3. Now using the expression for $I I^{5}$

$$
I I=-\frac{1}{N} \sum_{s \neq s^{\prime}}\left(\wp-\zeta^{2}\right)\left(q_{s}-q_{s^{\prime}}\right),
$$

we will estimate

$$
\begin{aligned}
I+I I= & \frac{\sigma^{2}}{N^{2}} K_{N}-\frac{1}{2 N^{2}} \sum_{n=1}^{N}\left(\sum_{s=1}^{N} 2 \zeta\left(q_{n}-q_{s}\right)\right)^{2}-\frac{\sigma^{2}}{2 N^{3}} P_{N}^{2} \\
& -\frac{1}{N} \sum_{s \neq s^{\prime}} \wp\left(q_{s}-q_{s^{\prime}}\right)+\frac{1}{N} \sum_{s \neq s^{\prime}} \zeta^{2}\left(q_{s}-q_{s^{\prime}}\right) .
\end{aligned}
$$

${ }^{5}$ In the rational case $\wp-\zeta^{2} \equiv 0 ; L^{(1)}$ and $I I$ vanish identically. 
We will prove that

$$
\begin{aligned}
& -\frac{1}{2 N^{2}} \sum_{n=1}^{N}\left(\sum_{s=1}^{N} 2 \zeta\left(q_{n}-q_{s}\right)\right)^{2}+\frac{1}{N} \sum_{s \neq s^{\prime}} \zeta^{2}\left(q_{s}-q_{s^{\prime}}\right) \\
& =\frac{N-2}{N^{2}} \sum_{s \neq s^{\prime}} \wp\left(q_{s}-q_{s^{\prime}}\right) .
\end{aligned}
$$

This would imply

$$
I+I I=\frac{\sigma^{2}}{N^{2}} K_{N}-\frac{1}{N^{2}} V_{N}-\frac{\sigma^{2}}{2 N^{3}} P_{N}^{2}
$$

and complete the proof of the theorem.

Step 4. To prove the identity (7) we make some manipulations with the sums

$$
\begin{aligned}
& -\frac{2}{N^{2}} \sum_{n=1}^{N}\left[\sum_{s=1}^{N} \zeta\left(q_{n}-q_{s}\right)\right]^{2}+\frac{1}{N} \sum_{s \neq s^{\prime}} \zeta^{2}\left(q_{s}-q_{s^{\prime}}\right) \\
& =-\frac{2}{N^{2}} \sum_{s \neq n, s^{\prime} \neq n} \zeta\left(q_{n}-q_{s}\right) \zeta\left(q_{n}-q_{s^{\prime}}\right)+\frac{1}{N} \sum_{s \neq s^{\prime}} \zeta^{2}\left(q_{s}-q_{s^{\prime}}\right) \\
& =\frac{1}{N^{2}}\left[(N-2) \sum_{s \neq s^{\prime}} \zeta^{2}\left(q_{s}-q_{s^{\prime}}\right)-2 \sum_{s \neq s^{\prime} \neq n} \zeta\left(q_{n}-q_{s}\right) \zeta\left(q_{n}-q_{s^{\prime}}\right)\right] \\
& =\frac{1}{N^{2}}\left[\sum_{s \neq s^{\prime} \neq n} \zeta^{2}\left(q_{s}-q_{s^{\prime}}\right)+2 \zeta\left(q_{s}-q_{n}\right) \zeta\left(q_{n}-q_{s^{\prime}}\right)\right] .
\end{aligned}
$$

Fix $k_{1}<k_{2}<k_{3}$ and consider all their permutations. Collect the terms with such indices

$$
\begin{aligned}
& \frac{2}{N^{2}} \sum_{k_{1}<k_{2}<k_{3}} \zeta^{2}\left(q_{k_{1}}-q_{k_{2}}\right)+\zeta^{2}\left(q_{k_{2}}-q_{k_{3}}\right)+\zeta^{2}\left(q_{k_{3}}-q_{k_{1}}\right) \\
& \quad+2 \zeta\left(q_{k_{1}}-q_{k_{3}}\right) \zeta\left(q_{k_{3}}-q_{k_{2}}\right)+2 \zeta\left(q_{k_{1}}-q_{k_{2}}\right) \zeta\left(q_{k_{2}}-q_{k_{3}}\right) \\
& \quad+2 \zeta\left(q_{k_{2}}-q_{k_{1}}\right) \zeta\left(q_{k_{1}}-q_{k_{3}}\right) \\
& =\frac{2}{N^{2}} \sum_{k_{1}<k_{2}<k_{3}}\left(\zeta\left(q_{k_{1}}-q_{k_{2}}\right)+\zeta\left(q_{k_{2}}-q_{k_{3}}\right)+\zeta\left(q_{k_{3}}-q_{k_{1}}\right)\right)^{2} .
\end{aligned}
$$

Using the identity

$$
(\zeta(u)+\zeta(v)+\zeta(s))^{2}=\wp(u)+\wp(v)+\wp(s)
$$

for $u+v+s=0$, we obtain

$$
\frac{2}{N^{2}} \sum_{k_{1}<k_{2}<k_{3}} \wp\left(q_{k_{1}}-q_{k_{2}}\right)+\wp\left(q_{k_{2}}-q_{k_{3}}\right)+\wp\left(q_{k_{3}}-q_{k_{1}}\right) .
$$


The last expression is invariant under permutations and therefore it is equal

$$
\begin{aligned}
& \frac{2}{6 N^{2}} \sum_{s \neq s^{\prime} \neq n} \wp\left(q_{s}-q_{s^{\prime}}\right)+\wp\left(q_{s}-q_{n}\right)+\wp\left(q_{s^{\prime}}-q_{n}\right) \\
& =\frac{N-2}{N^{2}} \sum_{s \neq s^{\prime}} \wp\left(q_{s}-q_{s^{\prime}}\right) .
\end{aligned}
$$

This completes the proof of the identity (7) and the theorem.

Example. For $N=2$ the equation defining the curve $\Gamma_{2}$ has the form

$$
k^{2}+k \frac{\sigma P}{2}+\left(\frac{\sigma^{2} P^{2}}{8}-\frac{\sigma^{2} H}{4}-\wp(z)\right)=0 .
$$

Solving the quadratic equation

$$
k_{1}(z)=-\frac{\sigma P}{4}+\frac{1}{2} \sqrt{\frac{\sigma^{2} P^{2}}{4}-4\left(\frac{\sigma^{2} P^{2}}{8}-\frac{\sigma^{2} H}{4}-\wp(z)\right)} .
$$

Expanding $k_{1}(z)$ at $z=0$ we obtain

$$
k_{1}(z)=\frac{1}{z}-\frac{\sigma P}{4}+z\left(\frac{\sigma^{2} H}{8}-\frac{\sigma^{2} P^{2}}{32}\right)+O\left(z^{2}\right) .
$$

\section{Trace formula.}

If the total momentum vanishes, $P_{N}=0$, then the result of Theorem 9 becomes

$$
k_{1}(z)=\frac{N-1}{z}+z \frac{\sigma^{2} H_{N}}{2 N^{2}}+O\left(z^{2}\right) .
$$

Theorem 11. The following identity holds

$$
\frac{\sigma^{2}}{2 N^{2}} H_{N}=\sum_{\alpha=2}^{N} I_{\alpha}^{\prime}
$$

where $I_{\alpha}^{\prime}=-k_{\alpha}^{(1)}$.

$\sigma=i$. The antiinvolution $\tau_{i}$ does not permute the sheets of the curve. The variables $I_{\alpha}^{\prime}$ are real for all configurations of particles.

$\sigma=1$. For some configurations of particles the antiinvolution $\tau_{1}$ permutes some lower sheets $\alpha$ and $\tau_{1} \alpha$. It also leaves the other sheets invariant. Variables $I_{\alpha}^{\prime}$ and $I_{\tau_{1} \alpha}^{\prime}$ corresponding permuting sheets form complex conjugate paires. All other I's corresponding invariant sheets are real.

Proof. By Cauchy's theorem

$$
\frac{1}{2 \pi i} \int_{\gamma_{1}} k(P) \wp(z(P)) d z(P)=-\sum_{\alpha=2}^{N} \frac{1}{2 \pi i} \int_{\gamma_{\alpha}} k(P) \wp(z(P)) d z(P),
$$


where $\gamma_{\alpha}$ is a small contour surrounding $P_{\alpha}$, the point on the $\alpha$ 'th sheet above $z=0$. The asymptotics (8) implies the result.

The second part of the proof is different for repulsive and attractive cases. We treat them separately.

Repulsive case. $\sigma=i$. If the pair $(k, z)$ satisfies

$$
k(z)=\frac{1}{z} k_{\alpha}^{(-1)}+k_{\alpha}^{(0)}+k_{\alpha}^{(1)} z+\cdots
$$

in the vicinity of $P_{\alpha}$, then the pair $(-\bar{k},-\bar{z})$ also satisfies similar expression in the vicinity of $P_{\tau_{i} \alpha}$

$$
-\bar{k}(z)=\frac{1}{-\bar{z}} k_{\tau_{i} \alpha}^{(-1)}+k_{\tau_{i} \alpha}^{(0)}+k_{\tau_{i} \alpha}^{(1)}(-\bar{z})+\cdots .
$$

Therefore

$$
k(z)=\frac{1}{z} \bar{k}_{\tau_{i} \alpha}^{(-1)}-\bar{k}_{\tau_{i} \alpha}^{(0)}+\bar{k}_{\tau_{i} \alpha}^{(1)} z-\cdots .
$$

Comparision shows that $k_{\alpha}^{(n)}=\bar{k}_{\tau_{i} \alpha}^{(n)}$ for $n$ odd and $k_{\alpha}^{(n)}=-\bar{k}_{\tau_{i} \alpha}^{(n)}$ for $n$ even.

We know that $k_{1}^{(-1)}=N-1$ and $k_{\alpha}^{(-1)}=-1$ for $\alpha=2, \ldots, N$ so that $\tau_{i}$ leaves the upper sheet invariant.

The Lemma 5 states that the values of $k_{\alpha}^{(0)}$ are distinct on different sheets of the curve for a generic configuration of perticles. The matrix $\left(L^{(0)} a_{\alpha}^{(0)}, a_{\alpha^{\prime}}^{(0)}\right.$ ) (see the proof of Lemma 5) is skew symmetric and therefore all $k_{\alpha}^{(0)}$ are pure imaginary: $\bar{k}_{\alpha}^{(0)}=-k_{\alpha}^{(0)}$. These together with the identity $\bar{k}_{\alpha}^{(0)}=-k_{\tau_{i} \alpha}^{(0)}$ imply that $\tau_{i} \alpha=\alpha$ and antiinvolution $\tau_{i}$ does not permute sheets. Therefore $k_{\alpha}^{(1)}=\bar{k}_{\alpha}^{(1)}$ and approximation arguments complete the proof.

Attractive case. $\quad \sigma=1$. Arguments as before lead to $k_{\alpha}^{(n)}=\bar{k}_{\tau_{1} \alpha}^{(n)}$ for all $n$. Skew-symmetry of the matrix $L^{(0)}$ is lost but for generic configuration of particles $k_{\alpha}^{(0)}$ are distinct. The antiinvolution $\tau_{1}$ leaves the upper sheet invariant, but is can permute the lower sheets of the curve. An example after the proof of the Theorem shows how it happens for $N=3$. Therefore, some lower sheets $\alpha$ and $\tau_{1} \alpha$ are interchanged by antiinvolution $\tau_{1}$ and some are invariant. These and $k_{\alpha}^{(-1)}=\bar{k}_{\tau_{1} \alpha}^{(-1)}$, together with approximation arguments complete the proof of Theorem.

Example. For $N=3$ we can explicitly compute expansion for $k_{\alpha}(z)$ at $z=0$. In this case

$$
R_{3}(k, z)=8 k^{3}+4 \sigma P k^{2}+\left[\sigma^{2}\left(P^{2}-2 H\right)-24 \wp(z)\right] k+8 \sigma J+8 \wp^{\prime}(z),
$$

where

$$
8 J=\sigma^{2} p_{1} p_{2} p_{3}+4 p_{1} \wp\left(q_{2}-q_{3}\right)+4 p_{2} \wp\left(q_{1}-q_{3}\right)+4 p_{3} \wp\left(q_{1}-q_{2}\right) .
$$


If $P=0$ the equation of the curve $\Gamma_{3}$ becomes

$$
k^{3}-k\left[\frac{\sigma^{2} H}{4}+3 \wp(z)\right]+\sigma J+\wp^{\prime}(z)=0 .
$$

Cardano's formula for the roots

$$
k^{3}+p k-q=0
$$

has the form

$$
\begin{aligned}
& k_{1}(z)=\sqrt[3]{\frac{q}{2}+\sqrt{R}}+\sqrt[3]{\frac{q}{2}-\sqrt{R}}, \\
& k_{2}(z)=\omega^{2} \sqrt[3]{\frac{q}{2}+\sqrt{R}}+\omega \sqrt[3]{\frac{q}{2}-\sqrt{R}} \\
& k_{3}(z)=\omega \sqrt[3]{\frac{q}{2}+\sqrt{R}}+\omega^{2} \sqrt[3]{\frac{q}{2}-\sqrt{R}}
\end{aligned}
$$

where $\omega=e^{\frac{2 \pi i}{3}}, R=\frac{q^{2}}{4}+\frac{p^{3}}{27}$. After elementary but tiresome calculations we obtain

$$
\begin{aligned}
& k_{1}(z)=\frac{2}{z}+z \frac{\sigma^{2} H}{18}+O\left(z^{2}\right), \\
& k_{2}(z)=-\frac{1}{z}+\frac{\sigma \sqrt{3 H}}{6}+z\left(\frac{J}{\sqrt{3 H}}-\frac{\sigma^{2} H}{36}\right)+O\left(z^{2}\right), \\
& k_{3}(z)=-\frac{1}{z}-\frac{\sigma \sqrt{3 H}}{6}+z\left(-\frac{J}{\sqrt{3 H}}-\frac{\sigma^{2} H}{36}\right)+O\left(z^{2}\right) .
\end{aligned}
$$

Consider the case $\sigma=1$. If $H<0$, then $\tau_{1}$ permutes lower sheets of the curve $\Gamma_{3} ; I_{2}$ and $I_{3}$ are complex conjugate. If $H>0$, then $\tau_{1}$ leaves lower sheets invariant; $I_{2}$ and $I_{3}$ are real.

Lemma 12. Let $p_{n}=0, n=1, \ldots, N$. Then the curve $\Gamma_{N}$ admits involution

$$
\tau_{-}:(k, z) \rightarrow(-k,-z) .
$$

The involution $\tau_{-}$leaves the upper sheet invariant and $k_{1}^{(n)}=0$ for $N$ even. It can permute lower sheets and in this case $k_{\alpha}^{(n)}=k_{\tau_{-\alpha}}^{(n)}$ for $n$ odd and $k_{\alpha}^{(n)}=-k_{\tau_{-} \alpha}^{(n)}$ for $n$ even.

$$
\sigma=1 \text {. All } k_{\alpha}^{(0)}, \alpha=2, \ldots, N \text { are pure imaginary. }
$$

Proof. Using the identity $\Phi(q,-z)=-\Phi(-q, z)$ we have

$$
L(q, p=0,-z)+2(-k)=[L(q, p=0, z)+2 k]^{T}(-1)^{N} .
$$

Therefore $R(-k,-z)=(-1)^{N} R(k, z)$ and the existence of $\tau_{-}$is proved.

If a pair $(k, z)$ satisfies

$$
k=\frac{1}{z} k_{\alpha}^{(-1)}+k_{\alpha}^{(0)}+k_{\alpha}^{(1)} z+\cdots .
$$


Then

$$
-k=\frac{1}{-z} k_{\tau_{-\alpha}}^{(-1)}+k_{\tau_{-\alpha}}^{(0)}+k_{\tau_{-} \alpha}^{(1)}(-z)+\cdots .
$$

Therefore $k_{\alpha}^{(n)}=k_{\tau_{-} \alpha}^{(n)}$ for $\mathrm{n}$ odd and $k_{\alpha}^{(n)}=-k_{\tau_{-\alpha}}^{(n)}$ for $n$ even. Since $k_{1}^{(-1)}=$ $N-1$ and $k_{\alpha}^{(0)}=-1, \alpha=2, \ldots, N ; \tau_{-}$leaves the upper sheet invariant. The involution $\tau_{-}$can permute the lower sheets. It is demonstrated by the example for $N=3$.

$\sigma=1$. Skew-selfadjointness of the matrix $L^{(0)}$ is restored under the conditions of the Lemma.

\section{Appendix.}

The Weierstrass $\sigma(z)$ has periods $2 \omega$ and $2 \omega^{\prime}$ and is defined as

$$
\sigma(z)=z \prod\left\{\left(1-\frac{z}{\boldsymbol{\omega}}\right) \exp \left(\frac{z}{\boldsymbol{\omega}}+\frac{z^{2}}{2 \boldsymbol{\omega}^{2}}\right)\right\}
$$

where $\boldsymbol{\omega}=2 \omega n+2 \omega^{\prime} n^{\prime}$ and $\prod$ is taken with $n, n^{\prime} \in \mathbb{Z}^{1} ; n^{2}+n^{\prime 2}>0$. $\zeta(z)$ and $\wp(z)$ are difined similarly:

$$
\zeta(z)=\frac{d}{d z} \log \sigma(z), \quad \wp(z)=-\frac{d}{d z} \zeta(z) .
$$

For more information see $[\mathbf{H C}]$.

Acknowledgments. Finally, I would like to thank I.M. Krichever and H.P. McKean for numerous stimulating discussions.

\section{References}

[AMM] H. Airault, H.P. McKean and J. Moser, Rational and elliptic solutions of the Korteweg-de Vries equation and a related many-body problem, Comm. Pure Appl. Math., 30 (1977), 95-148.

[HC] A. Hurwitz and R. Courant, Allgemeine funktionentheorie und elliptische functionen, Springer-Verlag, Berlin-Gottingen-Heidelberg-New York, 1964.

[K] I.M. Krichever, Elliptic solutions of the Kadomcev-Petviasvili equations and integrable systems of particles, Funktsional. Anal. i Prilozhen, 14 (1980), 45-54.

[KBBT] I.M. Krichever, O. Babelon, E. Billey and M. Talon, Spin generalization of Calogero-Moser system and the matrix KP equation, in 'Topics in topology and mathematical physics', (1995), 83-119; Amer. Math. Soc. Transl., 170, Amer. Math. Soc.

[KNS] V.B. Kuznetsov, F.W. Nijhoff and E.K. Sklyanin, Separation of variables for the Ruijsenaars system, Comm. Math. Phys., 189 (1997), 855-877.

[MCV1] H.P. McKean and K.L. Vaninsky, Action-angle variables for nonlinear Schrödinger equation, Comm. Pure Appl. Math., 50 (1997), 489-562.

[MCV2] _ Cubic Schrödinger: The petit canonical ensemble in action-angle variables, Comm. Pure Appl. Math., 50 (1997), 593-622. 
[V1] K.L. Vaninsky, Gibbs states for Moser-Calogero potentials, International Journal of Modern Physics, B 11 (1997), 203-211.

[V2] Symplectic structures and volume elements in the function space for the cubic Schrödinger equation, Duke Math. J., 92(2) (1998), 381-402.

Received September 25, 1997 and revised May 1, 1998. The work is supported by NSF grant DMS-9501002.

Kansas State University

Manhattan, KS 66502

E-mail address: vaninsky@math.ksu.edu 